\title{
Investigation of the near-grazing behavior in hard-impact oscillators using model-based TS fuzzy approach
}

\author{
Kamyar Mehran · Bashar Zahawi · Damian Giaouris
}

Received: date / Accepted: date

\begin{abstract}
An impact oscillator is a non-smooth dynamical system with discontinuous state jumps whose dynamical behavior illustrates a variety of nonlinear phenomena including a grazing bifurcation. This specific phenomenon is difficult to analyze because it coincides with an infinite stretching of the phase space in the neighborhood of the grazing orbit, resulting in the well-known problem of the square-root singularity of the Jacobian of the discrete-time map. A novel TakagiSugeno fuzzy model-based approach is presented in this paper to model a hard impacting system as a nonsmooth dynamical system including discontinuous jumps. Employing non-smooth Lyapunov theory, the structural stability of the system is analyzed to predict the onset of the destabilizing chaotic behavior. The proposed stability results, formulated as a Linear Matrix Inequality (LMI) problem, demonstrate how the new method can detect the loss of stability just before the grazing bifurcation.
\end{abstract}

Keywords Takagi-Sugeno fuzzy approach · nonsmooth Lyapunov theory · Linear Matrix Inequality . Impact oscillator · Grazing

Kamyar Mehran

School of Electrical, Electronic and Computer Engineering, Newcastle University, NE1 7RU, England, UK.

E-mail: kamyar.mehran@ncl.ac.uk

Bashar Zahawi

School of Electrical, Electronic and Computer Engineering, Newcastle University, NE1 7RU, England, UK.

Damian Giaouris

School of Electrical, Electronic and Computer Engineering, Newcastle University, NE1 7RU, England, UK.

\section{Introduction}

Impacting systems are non-smooth dynamical systems in which components may experience impacts. Many experiments have been conducted on the dynamics of systems whose numerical models interchange between continuous and discrete states $[12,15,21,33,42]$. Strong nonlinear behavior, induced by rigid collisions, has been observed in most cases driving these systems into chaos [11]. Their unique complex dynamics include a periodic orbit instantly losing its stability to a chaotic vibration as a system parameter is varied. When a tangential grazing impact occurs between the system components, a unique phenomena known as a grazing bifurcation effectuates a local (infinite) stretching of phase space, which in turn has an intense destabilizing effect on system dynamics $[11,28,29]$. The dynamics of the impact oscillator have been the subject of intense interest in the literature. From the mathematical modeling point of view, impacting systems are categorized as a significant sub-group of non-smooth systems mainly because of the presence of discontinuous state jumps in their dynamics. The overall dynamics of such systems can be represented by a series of smooth functions, interrupted by discontinuous velocity reversals, making their maps non-smooth along the switching manifold $[11,30]$. From the stability analysis point of view, the square-root singularity in their Poincaré map results in a sudden change from a stable period-one behavior to an unstable, large chaotic attractor $[28,29]$. The sudden change is excited by a grazing condition where the square-root term of the Jacobian of the Poincaré map at the grazing point takes an infinite value, destabilizing the system as more impacts occur and its dynamical equations become more complex. 
The predominant analytical tool for the study of nonlinearities in impacting systems is discontinuity mapping $[11,28,29]$. This approach enables the analysis of grazing bifurcations involving limit cycles and other complex invariant sets encompassing more than equilibria. The discontinuity map, a term first coined by Nordmark [28], is a synthesized Poincaré map which is piece-wise smooth and defined locally near the grazing point at which a trajectory interacts with a discontinuity boundary. The technique has been popular for studying the stability of impacting systems especially when used in combination with a global Poincaré map derived around the limit cycle of interest. In this case, one can typically derive a non-smooth map whose orbits fully describe the dynamics in question [11]. Other attempts using explicit mapping to analyze mechanical impacting systems have also been published $[7,31]$. In all cases, however, disagreements have been observed between the theoretical analysis employing the above methods and various experimental studies $[12,14,42$, 43]. More recently, numerical analysis of soft-impact oscillators employing the discontinuity-mapping approach has been proposed to study the onset of the grazing bifurcation $[24,25]$ with results showing good agreement with experimental observations $[15,25]$. However, a number of limitations and difficulties, both in terms of modeling and stability analysis, still exist when attempting to apply the existing theory directly to hardimpacting systems as the square-root singularity problem causes the Jacobian of the discontinuity map to assume infinite values near the grazing orbit.

Since the publication of the seminal paper by Tanaka and Sugeno [39] on fuzzy model-based control, there has been a great deal of interest in modeling nonlinear systems as TS fuzzy systems and formulating their stability analysis as a Linear Matrix Inequality (LMI) problem. However this body of work has been dominated by the study of smooth dynamical systems $[2,6,38,40,41]$ rather than non-smooth nonlinear systems whose righthand sides are mathematically discontinuous. In a previous study, the authors proposed a TS fuzzy modeling structure for modeling non-smooth systems with a degree of smoothness ${ }^{1}$ (DoS) of one (Filippov-type systems) and successfully applied it to investigate the stability of an example non-smooth electronic converter using non-smooth Lyapunov theory $[26,27]$. This approach has proved to be effective in non-smooth function approximation and prediction of the edge of bifurcation phenomena in Filippov-type systems. The proposed non-smooth TS fuzzy modeling structure is further developed in this paper to include the discontin-

\footnotetext{
1 For a definition of the degree of smoothness of a nonsmooth system refer to [11].
}

uous state jump, a dynamical attribute of hard impact oscillators, i.e. non-smooth systems with the degree of smoothness (DoS) of zero. This is achieved by introducing an additional set of states in the TS fuzzy model structure. State jumps can then describe different discontinuous states, enabling the resulting model to represent all known complex nonlinear phenomena observed in hard impacting systems. Based on the proposed TS fuzzy model, the square-root singularity problem is then addressed by providing a Lyapunov framework for the stability analysis to investigate the sudden chaotic behavior in the impacting system. Although piece-wise Lyapunov functions have been used to analyze the stability of variable structured systems $[5,9$, $16,23]$ and even fuzzy systems employed for the modelbased stability of non-linear smooth functions [17], these previous studies have been all limited to the classical notion of stability (stability of equilibria). In this paper the stability analysis (based on non-smooth Lyapunov's method) introduced earlier by the authors to study nonsmooth Filippov systems is further developed for the examination of the stability of more complex invariant sets, i.e. periodic orbits of impacting systems, by recasting the stability conditions on Linear Matrix Inequalities (LMIs). In this way the resulting LMI conditions can accurately pinpoint the instability of the periodic orbit in question near the grazing point, which typically results in a discontinuity-induced bifurcation (DIB), i.e. a grazing bifurcation, immediately followed by abrupt chaos.

\section{The hard impact oscillator and its mathematical model}

In order to study the complex dynamics of impact oscillators, it suffices to consider a simple but archetypal model of a one-degree-of-freedom hard impacting system, as shown in Fig. 1. This simple impacting system is comprised of a hard wall positioned at a distance $\sigma$ from the center of a mass $M$ subject to an instantaneous impact with the wall. The state evolution of the system can be fully described by the position $s(t)$ and the velocity $v(t)=\frac{d s}{d t}=\dot{s}(t)$ of its center of mass. Assuming the presence of a linear spring and a dashpot that attach the mass to a datum point (Fig.1), the motion of impacts can be represented by a set of dimensionless differential equations [11]:

$\frac{d^{2} s}{d t^{2}}+2 \zeta \omega_{n} \frac{d s}{d t}+\omega_{n}^{2} s=g(t), \quad$ if $s>\sigma$.

where $\zeta$ is the damping factor and $\omega_{n}$ is the natural frequency of the oscillation. The mass and stiffness are set to unity, and $g(t)$ is an applied external force, which 


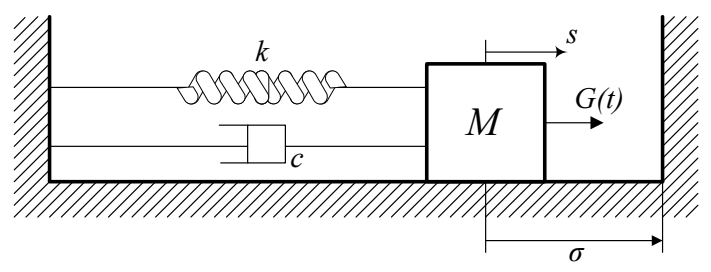

Fig. 1 A one-degree-of-freedom hard impact oscillator.

we consider as a periodic sinusoidal forcing function $g(t)=F \cos (w t)$ with a period of $T=2 \pi / w$ and an amplitude of $F$. It is of course possible to apply different types of forcing function $g(t)$ fed from an external flow or a solution of another problem [3]. However, in this paper, we consider a periodic sinusoidal forcing function to allow for an easier comparison of our results with previously published studies which were based on the nonlinear discontinuity mapping approach $[11,29,30]$. The system becomes highly nonlinear as a result of the instantaneous impacts. In the absence of impacts, the system (1) is linear and it's solution comprises exponentially decaying free oscillations converging to driven periodic motions at frequency $w$. For this impacting system (Fig. 1), $\zeta=c / 2 \sqrt{k M}, \omega_{n}=\sqrt{k / M}$ and $g(t)=G(t) / M$. If we assume a free-motion system, the impact with the rigid obstacle takes place at time $t_{0}$ at which $s=\sigma$, where $\sigma=0.5 \mathrm{~m}$. In fact, at $t=t_{0}$, motion and velocity just before the impact $\left(s\left(t_{0}\right), v\left(t_{0}\right)\right):=\left(s^{-}, v^{-}\right)$are mapped to the zero time motion and the zero time velocity just after the impact $\left(s^{+}, v^{+}\right)$as follows:

$s^{+}=s^{-} \quad$ and $\quad v^{+}=-r v^{-}$,

where $0<r<1$ is Newton's coefficient of restitution. The system is smooth in the region of $s<\sigma$ but at time $t=t_{0}$ there is a discontinuous state jump (velocity reversal) making the system (1) non-smooth. This can be analytically expressed assuming a discontinuity boundary $\Sigma$ (hard boundary $H(x)$ ) and a set of the reset map $R(x)$ by defining the system as $x=(s, \dot{s})^{T}$. Equations (1) and (2) can then be written as [11]:

$\dot{x}=F(x), \quad$ if $\quad x \in S^{+}$

$x \mapsto R(x), \quad$ if $\quad x \in \Sigma$

where, $S^{+}=\{x: H(x)>0\}$ and $\Sigma=\{x: H(x)=$ $0\} . H(x)$ is a smooth function which, in this case, is calculated as $H(x)=H(s, \dot{s})=s-\sigma$.

Fig. 2 [18] shows the different types of possible orbits near the discontinuity boundary including the solution trajectories corresponding to the grazing event (Fig. 2b). At the time of grazing, the solution trajectory is tangential to the discontinuity boundary $\Sigma$. After the impact (Fig. 2c) the system states instantly jump to a

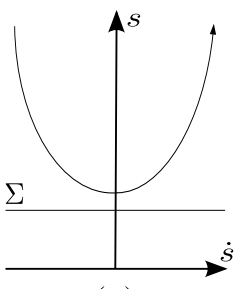

(a)

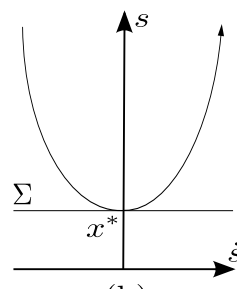

(b)

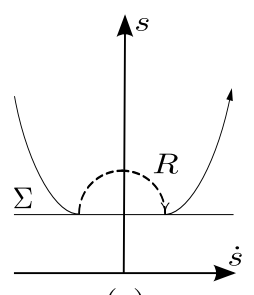

(c)
Fig. 2 The different types of orbit close to the discontinuity boundary $\Sigma$ : (a) a solution trajectory when there is no impact, (b) a solution trajectory at a grazing event, (c) a solution trajectory when impact occurs [18].

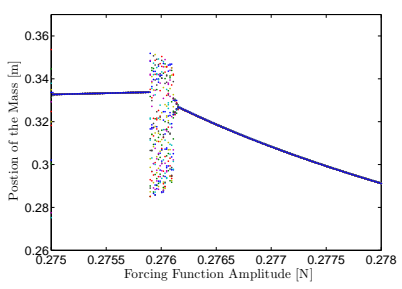

(a)

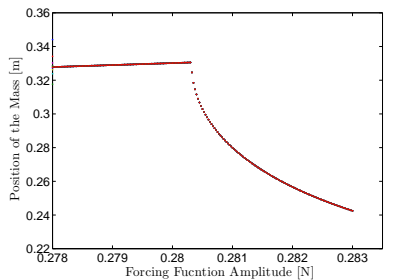

(b)

Fig. 3 (a) Typical grazing bifurcation diagram when a grazing event (Fig. 2b) occurs caused by infinite local stretching at $\varpi=2.97$. (b) The bifurcation diagram when the value of $\varpi$ is changed to $\varpi=3$. Here $\varpi$ represents the frequency ratio of the impacting system derived as $\varpi=\frac{2 \omega_{n}}{w_{\text {forcing }}} \sqrt{1-\zeta^{2}}$; $M=1 \mathrm{~kg}, c=0.5 \mathrm{~N}-\mathrm{s} / \mathrm{m}$, and $k=1 \mathrm{~N} / \mathrm{m}[18]$.

new position, given by the reset map $x \mapsto R(x)$. The effect of grazing (zero-velocity impact) can be strikingly destabilizing such that a stable periodic orbit is suddenly transformed to an unstable chaotic attractor as demonstrated in the bifurcation diagram of Fig. 3a. The bifurcation diagram of Fig. 3b shows the dynamics of the impact when there is still grazing but no abrupt chaotic orbit.

\section{Fuzzy Modeling of Hard Impacting Systems}

Generally, the nonlinear and multi-parametric nature of fuzzy control systems make their stability analysis a demanding task. To find a remedy, Takagi and Sugeno introduced a modeling approach in which a linear system can be adopted as the consequent part of a fuzzy rule, the so-called TS fuzzy model [35]. The TS model they proposed, elaborated in a number of consequent publications, is essentially described by a set of fuzzy implications, which characterize local relations of the 
system in state space. The main feature of a TS model is to express the local dynamics of each fuzzy rule (implication) by a linear state-space system model. The overall fuzzy system is then modeled by the fuzzy blending of these local linear system models through some suitable membership functions. As a mathematical expression, the $j^{\text {th }}$ rule of the continuous-time TS fuzzy model is formulated in the following form:

Plant Rule $j$ : IF $x_{1}(t)$ is $\Gamma_{1}^{j}$ AND...AND $x_{n}(t)$ is $\Gamma_{n}^{j}$

$$
\text { THEN } \dot{x}=A^{j} x(t)+B^{j} u(t), \quad j=1,2, \ldots, l,(5)
$$

where $\Gamma_{i}^{j}$ is a fuzzy set, $x(t) \in \Re^{n}$ is the state vector, $u(t) \in \Re^{m}$ is the control input vector, $A^{j} \in \Re^{n \times n}$ and $B^{j} \in \Re^{n \times m}$ are respectively the system matrix and the input matrix, and $l$ is the number of model rules. The defuzzified output of (5) is represented as:

$\dot{x}(t)=\sum_{j=1}^{l} \mu^{j}(x(t))\left(A^{j} x(t)+B^{j} u(t)\right)$,

where

$\omega^{j}(x(t))=\prod_{i=1}^{n} \Gamma_{i}^{j}\left(x_{i}(t)\right)$, and $\mu^{j}(x(t))=\frac{\omega^{j}(x(t))}{\sum_{k=1}^{l} \omega^{k}(x(t))}$

in which $\Gamma_{i}^{j}\left(x_{i}(t)\right)$ is the grade of membership of $x_{i}(t)$ in $\Gamma_{i}^{j}$. Basic properties of the weighting function $\omega^{j}(t)$ are: $\omega^{j}(x(t)) \geq 0$, and $\sum_{k=1}^{l} \omega^{k}(x(t))>0, k=1,2, \ldots, l$. It is clear that $\mu^{j}(x(t)) \geq 0$, and $\sum_{j=1}^{l} \mu^{j}(x(t))>0, j=$ $1,2, \ldots, l$.

A discrete-time TS fuzzy model may also be obtainable by following a similar procedure [36]. Other techniques to allow discretization of Linear time-varying (LTI) continuous-time TS fuzzy systems have also been discussed, e.g. [19]. However, regardless of the fuzzy approximation method, the TS fuzzy model described by (6) is only able to represent smooth dynamical systems to arbitrary accuracy $[26,27]$ and its mathematical structure is incapable of representing non-smooth dynamical equations ${ }^{2}$. As a solution, a synthesized TS fuzzy model which can incorporate discrete events to represent Filippov-type non-smooth systems has been proposed by the authors and successfully applied to DC-DC electronic converters [27]. In this section we will develop a TS fuzzy model capable of approximating hard impact oscillator systems to a high degree of accuracy to represent all observed nonlinear phenomena,

2 Non-smooth or piecewise-smooth systems are the terms initially coined for dynamical equations with a discontinuous right-hand side. However, they constitute different classes of systems (than initially defined by Filippov [13]) including flows and maps. Here we use the term non-smooth (dynamical) systems for non-smooth flows only as we limit our studies to this class. Refer to [11] for more detailed definitions and discussions. including the discontinuity-induced bifurcation (DIB) created near the grazing event (Fig. 3a). To enable

Table 1 Examples of mechanical non-smooth Filippov-type systems; dry-friction oscillator and mechanical impact system with discontinuous states. $S_{i}$ and $S_{j}$ are denoted as open regions of the phase space where system dynamics are respectively governed by $\dot{x}=F_{i}(x, \mu)$ and $\dot{x}=F_{j}(x, \mu) . \Sigma_{i j}$ is the discontinuity boundary or switching manifold.

\begin{tabular}{|c|c|c|}
\hline & dry friction & impact system \\
\hline ?2 & 1 & 0 \\
\hline 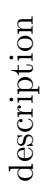 & $\begin{array}{l}\text { Non-smooth } \\
\text { Filippov-type } \\
\text { system with } \\
\text { sliding dynamics }\end{array}$ & $\begin{array}{c}\text { Non-smooth } \\
\text { system with } \\
\text { discontinuous(jump) } \\
\text { states }\end{array}$ \\
\hline 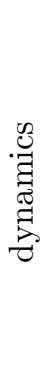 & $S_{i}$ & 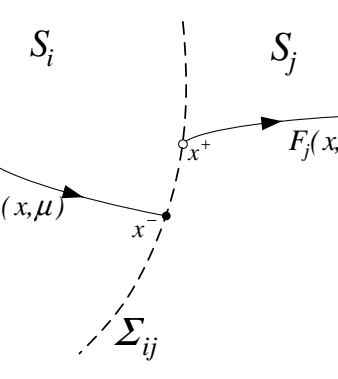 \\
\hline
\end{tabular}

the fuzzy model (6) to approximate both types of nonsmooth systems illustrated in Table $1^{3}$, two functions should be incorporated into the original TS fuzzy model (5) and (6). First, a function $\xi$ is composed to describe discrete events. This function, formally defined as $m^{+}(t)=\xi(x(t), m(t))$ where $m$ is a discrete state variable, describes interactions between the continuous and discrete states by determining the change in $m$ [27]. The fundamental function $\chi$ also needs to be defined to express any discontinuity (or jump) in system states, present in the dynamics of impact oscillator systems. From the formal definition of this function, $x^{+}(t)=\chi(x(t), m(t))$, it can be implied that $\chi$ explains the interaction between the discrete and discontinuous (or jump) states of the system, a property that cannot be found in Filippov-type non-smooth systems (Table 1). Accordingly, we propose a TS fuzzy model that can represent the impact oscillator system (1), (2) as a non-

3 Normally a sliding region in Filippov-type systems can be attracting or repelling. However, in case of a dry-friction oscillator where the system flow is forward in time, a repelling sliding region is not realizable in its sliding dynamics. 
smooth system (DoS of zero) as follows:

$$
\left\{\begin{array}{l}
\dot{x}=\sum_{j=1}^{l_{m}} \mu^{j}(x, m)\left(A^{j}(m) x+B^{j}(m) u\right) \\
m^{+}=\xi(x, m) \\
x^{+}=\chi(x, m)
\end{array}\right.
$$

where $x \in R^{n}$ is the continuous state, $m \in M=$ $\left\{m_{1}, \ldots, m_{N}\right\}$ is a discrete state $(N$ possibly infinite). The state space is the Cartesian product $\Re^{n} \times M$. Each local continuous dynamical function $f\left(x, m_{i}\right)$ is associated with a discrete state $m_{i} \in M$ and is represented by a specific set, which we will call a fuzzy sub-vector field, denoted by $F_{m_{i}}$ and described as:

$$
\begin{aligned}
F_{m_{i}}= & \sum_{\substack{j \in\{1,2, \ldots\}\\
}} \mu^{j}\left(x, m_{i}\right)\left(A^{j}\left(m_{i}\right) x+B^{j}\left(m_{i}\right) u\right), \\
& i \in I_{N}=\{1,2, \ldots, N\},
\end{aligned}
$$

where $A^{j}\left(m_{i}\right) \in \Re^{n \times n}, B^{j}\left(m_{i}\right) \in \Re^{n}$ and $\mu^{j}: \Re^{n} \times$ $M \rightarrow[01], i \in I_{l_{m}}$, are continuous weighting functions satisfying $\sum_{j=1}^{l_{m}} \mu^{j}(x, m)=1$ and $l_{m}$ is the number of fuzzy rules. The above representation includes the possibility of state discontinuities by defining $\chi$ : $\Re^{n} \times M \rightarrow \Re^{n}$, and the dynamics of discrete states by defining $\xi: \Re^{n} \times M \rightarrow M$. The notation $m^{+}$means the next state of $m$ (the event of switching from an active fuzzy sub-vector field to another) and $x^{+}$means the next state of $x$. The TS fuzzy system (7) is defined as an autonomous system where there are no external inputs influencing the dynamics. This may be the case when external inputs are feedback functions of the fuzzy continuous and discrete state.

Remark 1: Alternatively, the function $\xi$ may be described by a number of switch sets $S_{i, k}$, which are related to $\xi$ by

$S_{i, k}=\left\{x \in \Re^{n} \mid m_{k}=\xi\left(x, m_{i}\right)\right\}, i \in I_{N}, k \in I_{N}$,

where $I_{N}=\{1,2, \ldots, N\}$. Therefore, switch sets simply describe where in the continuous fuzzy state space a sub-vector field

$$
F_{m_{i}}=\sum_{j \in\{1,2, \ldots\}} w^{j}\left(x, m_{i}\right)\left(A^{j}\left(m_{i}\right) x+B^{j}\left(m_{i}\right) u\right)
$$

switches to another fuzzy sub-vector field

$$
F_{m_{k}}=\sum_{j \in\{1,2, \ldots\}} w^{j}\left(x, m_{k}\right)\left(A^{j}\left(m_{k}\right) x+B^{j}\left(m_{k}\right) u\right)
$$

on the condition that $m_{i} \neq m_{k}$. Depending on the application, the switch sets normally represent hypersurfaces or hyperplanes in phase space $\square$

Remark 2: Similarly, the function $\chi$ can be alternatively described by jump sets $D_{i}$, expressed as:

$D_{i}=\left\{x \in \Re^{n} \mid x^{+}=\chi\left(x, m_{i}\right)\right\}, i \in I_{N}$.
In practice, switch sets and jump sets coexist in space and time and the relation between two successive continuous fuzzy states can be defined by the matrix $J$ as

$x^{+}=J\left(m_{i}\right) x$.

In the following analysis, the matrix $J$ can be useful when applying zero-time mapping (2) to impact oscillators.

A non-smooth impacting system normally undergoes a discontinuous jump in the system state $x=R(x)$ at the switching manifold $\Sigma_{i j}$, and it has, as mentioned earlier, a DoS of zero. Even tough the local vector fields before and after the impact are equal $F_{1}(x)=F_{2}(x)$, there is a jump in the Jacobian derivatives $\frac{\partial F_{1}}{\partial x}$ and $\frac{\partial F_{2}}{\partial x}$ at $x$. Direct numerical simulation of the mathematical models of such systems is at best difficult. Different formalisms using specialist software platforms have been suggested in the literature to overcome these problems including complementarity systems developed as part of the SICONOS project $[11,32]$ and differential inclusions based on variational inequalities [1]. Examples of these specialist software packages include SLIDECONT, a tool developed as a driver for AUTO'97 by Dercole et al. [10] and specialized numerical routines developed by Nordmark et al. [30]. These high-cost, computationally expensive software platforms for non-smooth systems are still needed since the black-box integration routines employed by general-purpose softwares, e.g. MATLAB, assume a high degree of smoothness for the solution and cannot therefore tackle non-smooth dynamical equations.

From the simulation point of view, the main advantage of the proposed general TS fuzzy modeling structure (7) is its ability to be directly solved using widelyused integration routines already designed for smooth systems. However, to achieve this we first need to convert system equations (1) and (2) to a continuous TS fuzzy model as proposed by (7), and solve the equations as second-order ordinary differential equations. In this way, the local linear models before the impact $\left(F_{1}\right)$ and after the impact $\left(F_{2}\right)$ should be transformed into nonlinear models (due to the state transformation $x \mapsto x^{2}$ ), so that the Jacobian derivatives $\frac{\partial F_{1}}{\partial x}$ and $\frac{\partial F_{2}}{\partial x}$, can then be equal and linear. The nonlinear local models are then represented by the fuzzy sub-vector fields $F_{m_{i}}$ as suggested by (8). To further clarify this, we can describe the TS fuzzy formalism (7) in the form of differential inclusions $\dot{x} \in f(x)$, where

$$
f=\left\{\begin{array}{l}
\left\{F_{m_{1}}=\sum_{j=1}^{l} \mu^{j}\left(x, m_{1}\right)\left(A^{j}\left(m_{1}\right) x+B^{j}\left(m_{1}\right) u\right)\right\}, \text { if } x \in S_{1,2}, \\
\left\{F_{m_{2}}=\sum_{j=1}^{l} \mu^{j}\left(x, m_{2}\right)\left(A^{j}\left(m_{2}\right) x+B^{j}\left(m_{2}\right) u\right)\right\}, \text { if } x \in S_{2,1}, \\
\left\{F_{m_{1}}+\mu\left(F_{m_{2}}-F_{m_{1}}\right) \mid 0<\mu<1\right\}, \text { if } x \in \partial S_{1} \cap \partial S_{2} .
\end{array}\right.
$$


The resulting TS fuzzy model can then hold the existence property of an absolutely continuous solution at the point of discontinuity (or jump) as an approximative solution of the differential inclusion $f$. The third term of $f$ describing the discontinuous jump when crossing the switching manifold $\left(x \in \Sigma_{12}\right.$ or $x \in \partial S_{1} \cap \partial S_{2}$ ) would then hold naturally for the TS fuzzy approximation where the model is defined as a convex combination of the fuzzy sub-vector fields.

The model of the impact oscillator system (1), (2) can now be derived according to the general TS fuzzy model proposed in (7), (9) and (10) by the following fuzzy implications (rules):

Plant Rule $\boldsymbol{j}$ : IF $x_{2}(t)$ is $F^{j}$ THEN

$\dot{x}=\left\{\begin{array}{l}A^{j}\left(m_{i}\right) x(t)+B^{j}\left(m_{i}\right) u(t), \quad j=1,2, \quad i=1,2, \\ m^{+}=\xi(x, m), \\ x^{+}=\chi(x, m),\end{array}\right.$

where the state vector is defined as $x(t)=\left[x_{1}(t) x_{2}(t)\right]^{T}=$ $[s(t) \dot{s}(t)]^{T}$ and $F^{j}$ represent the fuzzy sets. The function $\xi$ is defined based on two discrete states $m_{1}$ and $m_{2}$ as follows:

$\xi: m_{1} \mapsto m_{2}$

$\xi: m_{2} \mapsto m_{1}$,

The interaction between the two states can then be expressed in terms of switch sets (9) as follows (see Remark 1):

$S_{1,2}=\left\{x \in R^{n} \mid x_{1}(t)-\sigma>0\right\}$,

$S_{2,1}=\left\{x \in R^{n} \mid x_{1}(t)-\sigma<0\right\}$.

To obtain the fuzzy sub-vector fields $F_{m_{1}}$ and $F_{m_{2}}$, which can switch if (14) holds, let's describe the original impact oscillator equation (1) in canonical form as follows:

$\left\{\begin{array}{l}\dot{x}_{1}=x_{2}, \\ \dot{x}_{2}=-\omega_{n}^{2} x_{1}-2 \zeta \omega_{n} x_{2}-F \cos (w t),\end{array}\right.$

where $s(t)=x_{1}(t)$ and $\dot{s}(t)=v(t)=x_{2}(t)$.

For constructing $F_{m_{1}}$, fuzzy set supports for the state variables $x_{1}$ and $x_{2}$ are respectively chosen as $x_{1}^{1} \in[-0.5,0.5]$ and $x_{2}^{1} \in\left[2 \hat{l}_{1}, 2 \hat{l}_{2}\right]$, where $\hat{l}_{1}=4.13$ and $\hat{l}_{2}=-3.72$ are the amplitude limits of the state $x_{2}$ while the system is structurally stable at $F=1.4975 \mathrm{~N}$, $\varpi=1$. If we assume two fuzzy variables $z_{1}=x_{1}$ and $z_{2}=x_{2}$, these can be described by fuzzy sets as follows:

$z_{1}=0.5 \cdot \Gamma^{1}\left(z_{1}\right)+(-0.5) \cdot \Gamma^{2}\left(z_{1}\right)$,

$z_{2}=8.26 \cdot \Gamma^{3}\left(z_{1}\right)+(-7.44) \cdot \Gamma^{4}\left(z_{1}\right)$.
Since $\Gamma^{1}\left(z_{1}\right)+\Gamma^{2}\left(z_{1}\right)=1$ and $\Gamma^{3}\left(z_{2}\right)+\Gamma^{4}\left(z_{2}\right)=1$, the membership functions are derived as:

$$
\begin{aligned}
& \Gamma^{1}\left(z_{1}\right)=\frac{1}{2}+z_{1}, \Gamma^{2}\left(z_{1}\right)=1-\Gamma^{1}\left(z_{1}\right), \\
& \Gamma^{3}\left(z_{2}\right)=\frac{1}{2}+\frac{z_{2}-0.4}{15.7}, \Gamma^{4}\left(z_{2}\right)=1-\Gamma^{3}\left(z_{2}\right) .
\end{aligned}
$$

Therefore the sub-system matrices for $F_{m_{1}}$ can be constructed as follows:

$$
\begin{aligned}
& A^{1}\left(m_{1}\right)=\left[\begin{array}{cc}
0 & 1 \\
\max _{z_{1} \in \Gamma^{1}} z_{1} \cdot\left(-\omega_{n}^{2}\right) \max _{z_{2} \in \Gamma^{3}} z_{2} \cdot\left(-2 \zeta \omega_{n}\right)
\end{array}\right], \\
& A^{2}\left(m_{1}\right)=\left[\begin{array}{cc}
0 & 1 \\
\max _{z_{1} \in \Gamma^{1}} z_{1} \cdot\left(-\omega_{n}^{2}\right) \max _{z_{2} \in \Gamma^{4}} z_{2} \cdot\left(-2 \zeta \omega_{n}\right)
\end{array}\right], \\
& A^{3}\left(m_{1}\right)=\left[\begin{array}{cc}
0 \\
\max _{z_{1} \in \Gamma^{2}} z_{1} \cdot\left(-\omega_{n}^{2}\right) \max _{z_{2} \in \Gamma^{3}} z_{2} \cdot\left(-2 \zeta \omega_{n}\right)
\end{array}\right], \\
& A^{4}\left(m_{1}\right)=\left[\begin{array}{c}
0 \\
\max _{z_{1} \in \Gamma^{2}} z_{1} \cdot\left(-\omega_{n}^{2}\right) \max _{z_{2} \in \Gamma^{4}} z_{2} \cdot\left(-2 \zeta \omega_{n}\right)
\end{array}\right], \\
& B^{1}\left(m_{1}\right)=B^{2}\left(m_{1}\right)=B^{3}\left(m_{1}\right)=B^{4}\left(m_{1}\right)=\left[\begin{array}{c}
0 \\
-F
\end{array}\right],
\end{aligned}
$$

giving the following matrices when one substitutes the respective numbers with $u(t)=\cos (w t)$ as the input signal:

$$
\begin{aligned}
& A^{1}\left(m_{1}\right)=\left[\begin{array}{cc}
0 & 1 \\
-0.5 \omega_{n}^{2}-(2 \cdot 8.26) \zeta \omega_{n}
\end{array}\right], \\
& A^{2}\left(m_{1}\right)=\left[\begin{array}{cc}
0 & 1 \\
-0.5 \omega_{n}^{2} & (2 \cdot 7.44) \zeta \omega_{n}
\end{array}\right], \\
& A^{3}\left(m_{1}\right)=\left[\begin{array}{cc}
0 & 1 \\
0.5 \omega_{n}^{2}-(2 \cdot 8.26) \zeta \omega_{n}
\end{array}\right], \\
& A^{4}\left(m_{1}\right)=\left[\begin{array}{cc}
0 & 1 \\
0.5 \omega_{n}^{2}(2 \cdot 7.44) \zeta \omega_{n}
\end{array}\right], \\
& B^{1}\left(m_{1}\right)=B^{2}\left(m_{1}\right)=B^{3}\left(m_{1}\right)=B^{4}\left(m_{1}\right)=\left[\begin{array}{c}
0 \\
-F
\end{array}\right],
\end{aligned}
$$

To construct the second fuzzy sub-vector filed $F_{m_{2}}$, fuzzy set supports for the state variables $x_{1}$ and $x_{2}$ are chosen as $x_{1}^{2} \in[-4,0.5]$ and $x_{2}^{2} \in[-7.32,8.086]$, respectively, giving the following sub-system matrices:

$$
\begin{aligned}
& A^{1}\left(m_{2}\right)=\left[\begin{array}{cc}
0 & 1 \\
-0.5 \omega_{n}^{2}-(2 \cdot 8.086) \zeta \omega_{n}
\end{array}\right], \\
& A^{2}\left(m_{2}\right)=\left[\begin{array}{cc}
0 & 1 \\
-0.5 \omega_{n}^{2} & (2 \cdot 7.32) \zeta \omega_{n}
\end{array}\right], \\
& A^{3}\left(m_{2}\right)=\left[\begin{array}{cc}
0 & 1 \\
4 \omega_{n}^{2}-(2 \cdot 8.086) \zeta \omega_{n}
\end{array}\right],
\end{aligned}
$$




$$
\begin{aligned}
& A^{4}\left(m_{2}\right)=\left[\begin{array}{cc}
0 & 1 \\
4 \omega_{n}^{2} & (2 \cdot 7.32) \zeta \omega_{n}
\end{array}\right], \\
& B^{1}\left(m_{2}\right)=B^{2}\left(m_{2}\right)=B^{3}\left(m_{2}\right)=B^{4}\left(m_{2}\right)=\left[\begin{array}{l}
0 \\
1
\end{array}\right] .
\end{aligned}
$$

Now, it just remains to introduce the discontinuous jump. This can be realized by defining the function $\chi$ in the model (12), which can alternatively be described by the jump sets $D_{i}$ in (10). Hence, the jump matrices $J\left(m_{1}\right)$ and $J\left(m_{2}\right)$, based on zero-time velocity mapping (2), can be derived as:

$J\left(m_{1}\right)=\left[\begin{array}{cc}1 & 0 \\ 0 & -r\end{array}\right], \quad J\left(m_{2}\right)=\left[\begin{array}{cc}1 & 0 \\ 0 & -1 / r\end{array}\right]$,

where the coefficient of restitution $r=0.9$.

The TS fuzzy model (12) is then used to study all the nonlinear phenomena discussed in section 2. To verify the accuracy of the proposed modeling method, the time responses of the original model (1), (2) and the proposed TS fuzzy model (12) with different forcing function amplitudes are compared (Fig. 4) showing very good agreement. Figs. $4 \mathrm{a}$ and $4 \mathrm{~b}$ show the time responses with both systems being stable at an excitation amplitude of $F=0.227 \mathrm{~N}$. In contrast, Figs. $4 \mathrm{c}$ and $4 \mathrm{~d}$ show the original and fuzzy systems operating in the chaotic region at $F=0.276 N$ while Figs. $4 \mathrm{e}$ and $4 \mathrm{f}$ show the first 40 s at a different excitation amplitude of $F=1.4975 \mathrm{~N}$. The initial $5 \mathrm{~s}$ time responses for $\varpi=1$, $F=1.4975 N$ are shown in Fig. 5 for three different initial conditions. The steady-state phase portraits of the system are also shown in Fig. 6. The qualitative behavior of the TS fuzzy model (12) can be best depicted in terms of bifurcation diagrams (Fig. 7), where at each fixed parameter value, a random number generator is used to select initial conditions for a range of different points within a suitably defined subset of the phase space. These results confirm the accuracy of the TS fuzzy model (12) when compared with the bifurcation diagrams of Fig. 3.

\section{Stability near the grazing point}

Stability analysis methods for smooth TS fuzzy systems ${ }^{4}$ are dominantly established around the classical notions of stability. Stability results, specially for chaotic systems, have been developed mainly through

\footnotetext{
4 From this point on, we will use the terminology smooth TS fuzzy system for describing TS fuzzy models with the well-known formalism of (5) and (6) capable of approximating smooth dynamical systems and the terminology non-smooth TS fuzzy system for describing TS fuzzy models capable of approximating non-smooth dynamical systems to an arbitrary accuracy as proposed by the general formalism (7).
}
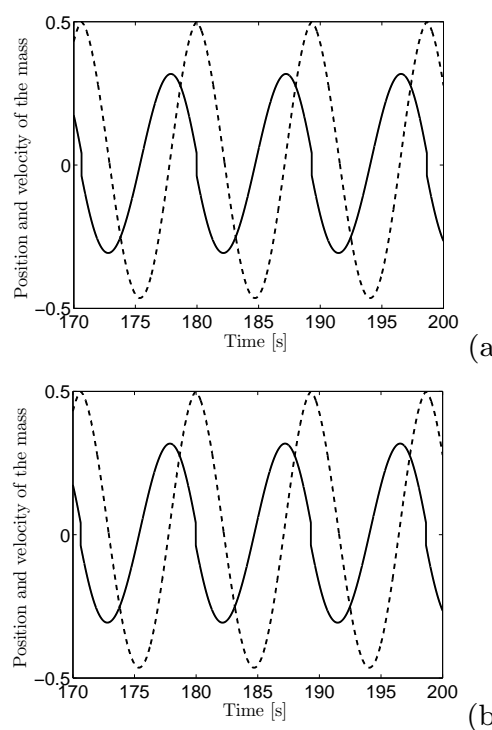

(a)

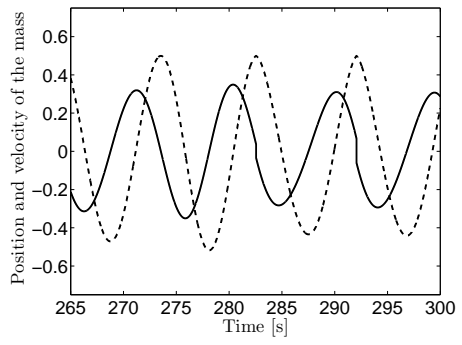

(b)
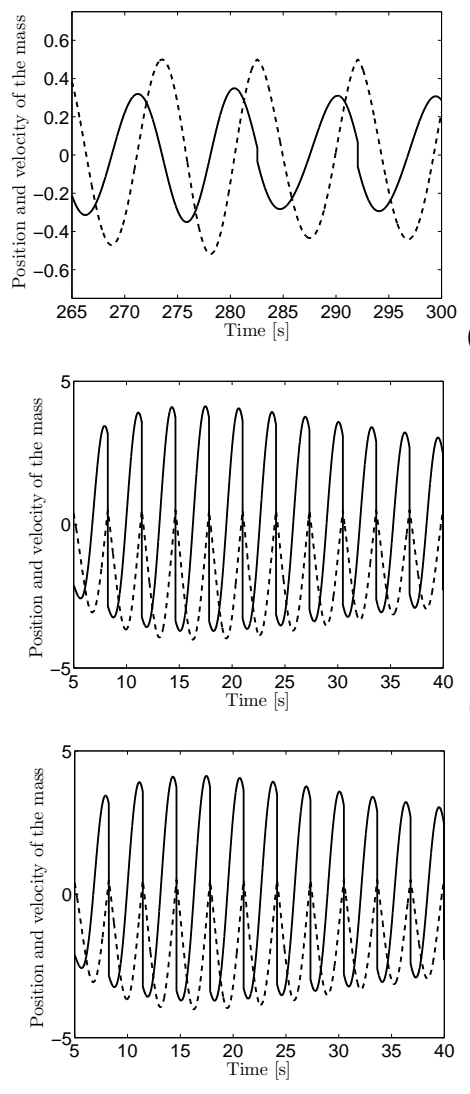

(e)

Fig. 4 Velocity and position responses with respect to time, produced from (a) the original impacting system $(\varpi=2.97$, $F=0.277 N)(\mathrm{b})$ the TS fuzzy model $(\varpi=2.97, F=0.277 N)$ (c) the original impacting system $(\varpi=2.97, F=0.276 N)$ (d) the TS fuzzy model $(\varpi=2.97, F=0.276 N)$ (e) the original impacting system $(\varpi=1, F=1.4975 N)$ (f) the TS fuzzy model $(\varpi=1, F=1.4975 N)$. 


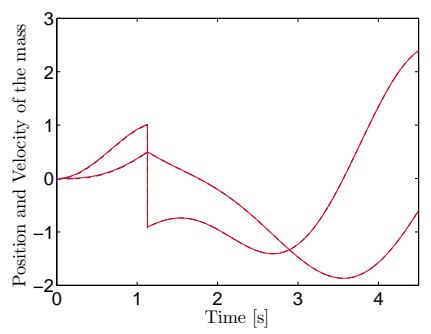

(a)

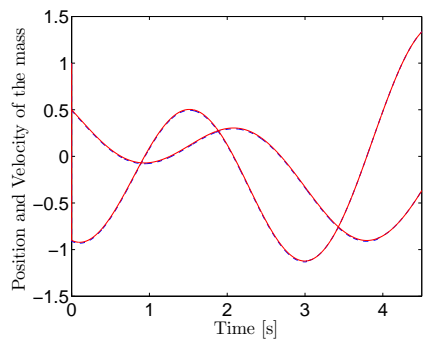

(b)

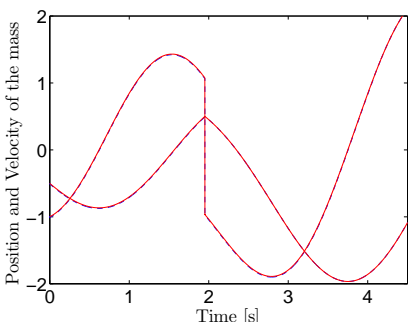

(c)

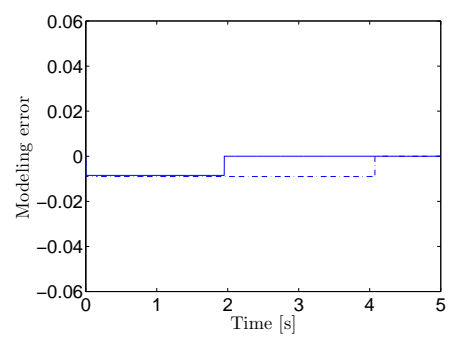

(d)

Fig. 5 Comparison of the TS fuzzy model responses (dashed line) with the original impacting system (solid line) for the operating condition $\varpi=1$ and $F=1.4975 N$ and initial conditions (a) $[00]^{T},(\mathrm{~b})[0.51]^{T}$, and (c) $[-0.5-1]^{T}$. Figure (d) shows the average initial error of the TS fuzzy model for both position (solid line) and velocity (dot-dashed line).

parallel-distributed compensation (PDC) technique to determine the rule structure of a TS fuzzy system and realized in terms of Lyapunov's direct method [36,37]. The derived stability frameworks are then formulated as linear matrix inequalities (LMIs) [36,37], which todate have proved to be the most efficient and systematic way to numerically search for convex constraints such as Lyapunov functions. The authors have already shown [27] that the formulation of the theorems based on smooth Lyapunov functions (see $[22,36]$ and the references therein) would end up in a conservative formulation even for smooth TS fuzzy systems. For nonsmooth cases, one may find the use of Lyapunov's method

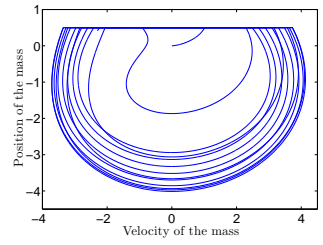

(a)
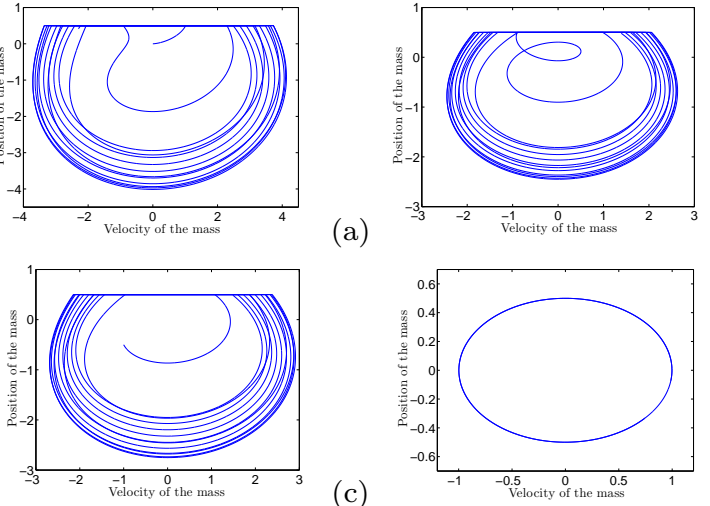

(b)

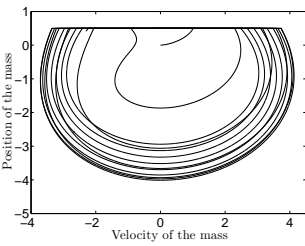

(c)

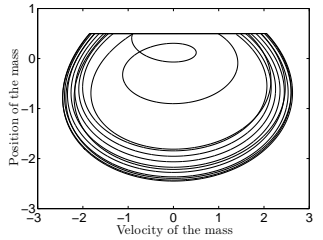

(d)

(e)
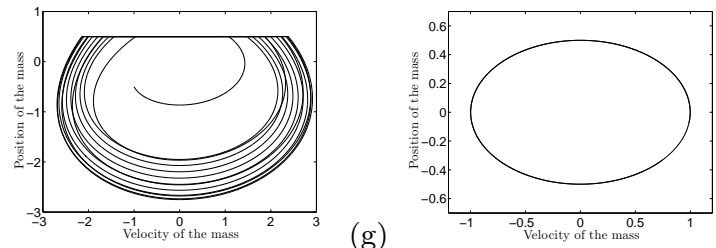

(f)

(g)

(h)

Fig. 6 Comparison of the phase portraits of the original system in (a), (b), (c), (d) with that of the TS fuzzy model (e), (f), (g), (h) for the operating condition $\varpi=1$ and $F=1.4975 \mathrm{~N}$ and initial conditions (a),(e) $\left[\begin{array}{ll}0 & 0\end{array}\right]^{T}$, (b),(f) $\left[\begin{array}{ll}0.5 & 1\end{array}\right]^{T}$, and $(\mathrm{c}),(\mathrm{g})[-0.5-1]^{T}$. Figures $(\mathrm{d})$ and $(\mathrm{h})$ show the steady-state responses (300s) when a stable limit cycle is observed.

to investigate the stability of equilibria very useful [11, 34]. However, if asymptotic stability is to be verified by finding a common Lyapunov function $V(x)$, which is positive definite and decreasing along the trajectories for each of the vector fields representing the system dynamics, no solution will be realizable (see $[11,34]$ and all references therein). It is therefore not surprising that there are mechanical non-smooth systems whose stability cannot be assessed using continuously differentiable Lyapunov theory $[11,20,34]$. Therefore, with a similar argument, the authors believe that any investigation of the structural stability of limit cycles in a non-smooth model, e.g. TS fuzzy model 12), should be based on discontinuous Lyapunov functions which are piece-wise smooth at the switching manifold. The fuzzy state space is allowed to be partitioned into relaxed regions of energy which are to be measured by local (Lyapunov) functions [27]. This is crucial in avoiding a conservative formulation of the resulting LMIs, which in turn, may lead to false stability results. The following steps are thus necessary in constructing an LMI framework 


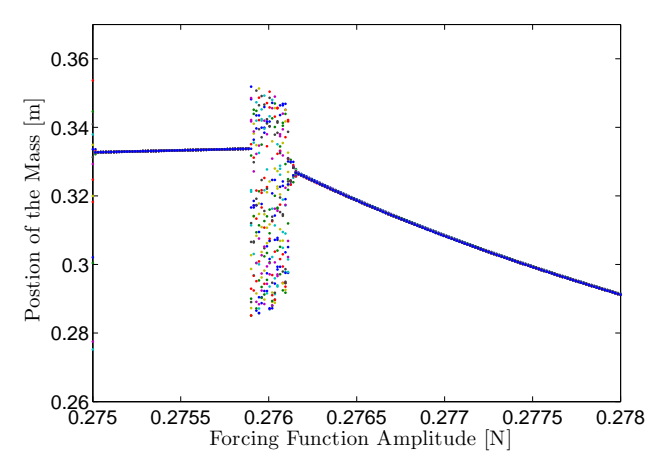

(a)

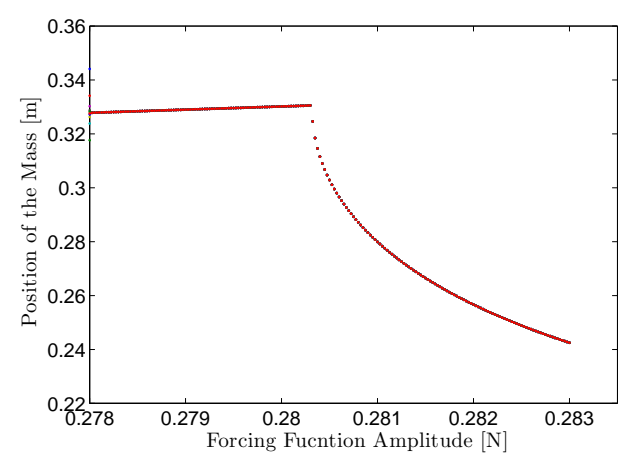

Fig. 7 The bifurcation diagrams produced from the proposed TS fuzzy model for (a) $\varpi=2.97$ when the grazing event makes the system chaotic (b) $\varpi=3$ when there is no sudden transition to chaotic behavior. The other common parameters are set as $M=1 \mathrm{~kg}, c=0.5 \mathrm{~N}-\mathrm{s} / \mathrm{m}$, and $k=1 \mathrm{~N} / \mathrm{m}$ (see Fig. 1).

for the bifurcation analysis of the non-smooth TS fuzzy model (12).

\subsection{Constructing piece-wise smooth Lyapunov functions}

If $\mathcal{F}$ is the fuzzy state space, a region $\Omega \subseteq \mathcal{F}$ is divided into $\Delta$ detached regions. It is assumed that the division is designed in such a way that if the trajectory starts at an initial point in region $\Omega, t_{k} k=1,2, \ldots$, it can only pass through to another detached region if the condition $t_{k}<t_{k+1}$ is satisfied. Let $\Omega$ be a set in $\mathcal{F}$ in which both continuous and discrete states are definable. The following subsets can then be defined:

$$
\begin{aligned}
\Omega^{x} & =\left\{x \in \Re^{n} \mid(x, m) \in \Omega\right\}, \\
\Omega^{x, m_{i}} & =\left\{x \in \Re^{n} \mid\left(x, m_{i}\right) \in \Omega\right\}, \\
\Omega^{m} & =\{m \in M \mid(x, m) \in \Omega\} .
\end{aligned}
$$

Continuous fuzzy states can be included in the sets $\Omega^{x}$ and $\Omega^{x, m_{i}}$ and discrete fuzzy states can be included in the set $\Omega^{m}$. If a trajectory fulfills the non-smooth TS fuzzy system with an initial fuzzy state $\left(x_{0}, m_{0}\right) \in \mathcal{F}_{0}$ [27] and we let $\varepsilon>0, q \in I_{\Delta}, r \in I_{\Delta}$, the regions $\Lambda_{q r}$ are defined as sets where the trajectory can pass from the region $\Omega_{q}$ to $\Omega_{r}$ by the following:

$$
\begin{gathered}
\Lambda_{q r}=\{(x, m) \in \Omega \mid \exists t>0 \text { such that }(x(t-\varepsilon), m(t-\varepsilon)) \\
\left.\in \Omega_{q} \text { and }(x(t+\varepsilon), m(t+\varepsilon)) \in \Omega_{r}, \text { when } \varepsilon \rightarrow 0\right\},
\end{gathered}
$$

which normally represents the hypersurfaces, i.e. switching manifolds $\Sigma_{i j}$. We also need to define another set to allow the trajectories to traverse from one region to another. Thus

$\mathrm{I}_{\Lambda}=\left\{(q, r) \mid \Lambda_{q r} \neq \emptyset\right\}$

is the set of tuples specifying the condition that there is at least one point for which the trajectory can traverse from $\Omega_{q}$ to $\Omega_{r}$.

Remark 3: The matrix $H\left(m_{i}\right), i \in I_{N}$ is defined as

$H\left(m_{i}\right)=\left[\begin{array}{cc}1 & 0 \\ r \frac{\partial H}{\partial x_{1}} & 1\end{array}\right]$,

where $r$ is the coefficient of restitution. The purpose of the above matrix is to impose a limit on the region boundary defined by the set $\Lambda_{q r}$ in (18). This matrix is used in the stability analysis presented in section 4.3 .

Let the overall Lyapunov function $V(x)$ be a discontinues function:

$V(x)=V_{q}(x)$ when $(x, m) \in \Omega_{q}$

where $V(x)$ is essentially non-smooth at the region boundaries, defined by $\Lambda_{q r}$, where $(q, r) \in \mathrm{I}_{\Lambda}$. The definition (19) is possible if we let $V_{q}: \mathbf{c l}^{5} \Omega_{q}^{x} \rightarrow \Re, V_{q}(x)=$ $\pi_{q}+2 p_{q}^{T} x+x^{T} P_{q} x, \pi_{q} \in \Re, p_{q} \in \Re^{n}, P_{q}=P_{q}^{T} \in$ $\Re^{n} \times \Re^{n}, q \in I_{\Delta}$ be a (scalar) quadratic function, locally continuous and Lyapunov in the local region $\Omega_{q}$. The local Lyapunov functions $V_{q}(x)$ can be defined in matrix form as:

$\tilde{P}_{q}=\left[\begin{array}{cc}P_{q} & p_{q} \\ p_{q}^{T} & \pi_{q}\end{array}\right]$,

where $\tilde{P}_{q}, q \in I_{\Delta}$ are piecewise quadratic matrices. Furthermore, the discontinuous Lyapunov function (19) is piecewise smooth with respect to time since the division (of regions) is made under the condition $t_{k}<t_{k+1}$ for every trajectory to pass through another region starting at an initial point in $\Omega$. Since $V_{q}(x)$ is defined as a continuous Lyapunov function in the region $\Omega_{q}$, its

${ }^{5}$ cl. denotes the closure of a set. 
derivative with respect to time using the sets (17) can be expressed as:

$$
\begin{gathered}
\dot{V}_{q}(x)=\sum_{j=1}^{l_{m}} w^{j}(\theta, m) \frac{\partial V_{q}(x)}{\partial t}\left(A^{j}(m) x+B^{j}(m)\right) \\
(x, m) \in \Omega_{q}^{x, m_{i}}, m_{i} \in \Omega_{q}^{m}
\end{gathered}
$$

4.2 Transforming confined conditions of regions to unconfined LMI conditions

All conditions of the stability theorem to be presented in section 4.3 should be confined to be fulfilled in part of the continuous fuzzy state space $\mathcal{F}$, which is divided into specific regions $\Omega_{q}^{x}, \Omega_{q}^{x, m_{i}}$ and $\Lambda_{q r}$. Before formulating any LMI condition, a description of how the confined stability conditions can be substituted by unconfined conditions is given here. This substitution can be made possible by first representing the regions by positive (quadratic) functions and then transforming to unconfined conditions using the general technique normally referred to as the $\mathcal{S}$ procedure [4]. In this section, the procedure is initially expounded in general terms and then applied to the confined conditions of the stability theorem.

Assume that $Q^{0}(x): \Re^{n} \rightarrow \Re$ is a function with unknown variables to be determined, fulfilling the condition

$Q_{0}(x) \geq 0$ for all $x$ in the region $\mathcal{R}$

Also assume that $Q_{k}(x): \Re^{n} \rightarrow \Re, k \in I_{s}$ are functions with known variables fulfilling the condition

$Q_{k}(x) \geq 0, k \in I_{s}$ for all $x$ in the region $\mathcal{R}$

Then, condition (21) can be substituted with more confined condition as follows:

$Q_{0}(x) \geq 0$ for all $x$ fulfilling $Q_{k}(x) \geq 0, k \in I_{s}$.

Condition (21) has thus been substituted by constraints represented by the functions $Q_{k}(x) \geq 0, k \in I_{s}$. This substitution is illustrated by Fig. 8. For formulating any stability condition to an LMI, the confined condition (22) should be substituted with an unconfined condition. This becomes possible by introducing additional variables $\delta_{k} \geq 0, k \in I_{s}$. Therefore, we initially have to consider the following Lemma:

Lemma [4]: Condition (22) holds if there exist $\delta_{k} \geq 0$ and $Q_{k}(x) \geq 0, k \in I_{s}$, such that

$\forall x \in \Re^{n}, Q_{0}(x) \geq \sum_{k=1}^{s} \delta_{k} Q_{k}(x)$.

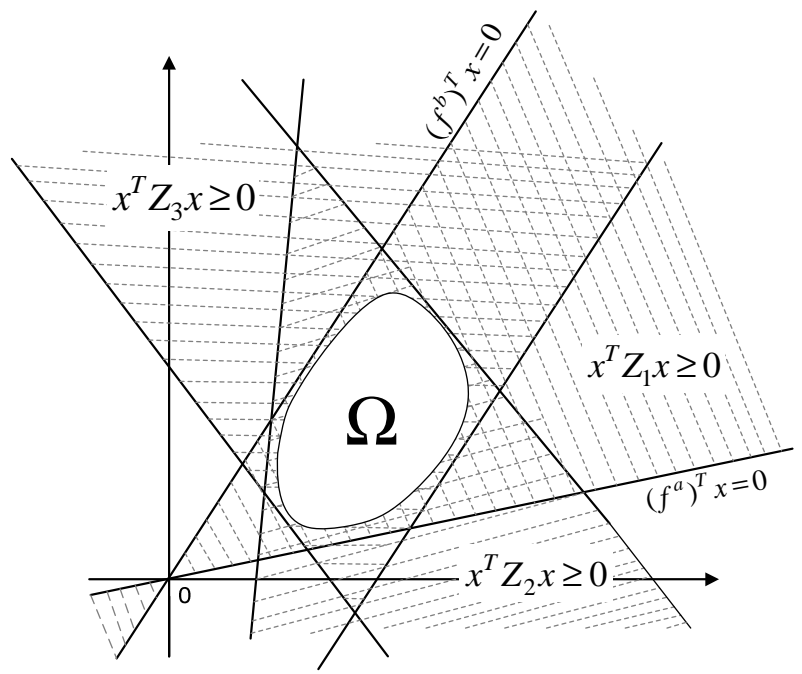

Fig. 8 The white region $\Omega$ is substituted with a region represented by semi-definite conditions $x^{T} Z_{1} x \geq 0, x^{T} Z_{2} x \geq 0$ and $x^{T} Z_{3} x \geq 0$, where each are limited by two hyperplanes $\left(f^{a}\right)^{T} x \geq 0$ and $\left(f^{b}\right)^{T} x \geq 0$.

The proof is easily attainable because we initially assume $\delta_{k} \geq 0, k \in I_{s}$ and $Q_{k}(x) \geq 0, k \in I_{s}$. Hence $\sum_{k=1}^{s} \delta_{k} Q_{k}(x) \geq 0$ for all $x$ fulfilling $Q_{k}(x) \geq 0, k \in I_{s}$.

The confined condition (21) can then be substituted by an unconfined condition using the above Lemma while defining the following quadratic functions:

$Q_{k}(x)=x^{T} Z_{k} x+2 c_{k}^{T} x+d_{k}, k=0, \ldots, s$,

where $Z_{k}=Z_{k}^{T} \in \Re^{n} \times \Re^{n}$ and $Q_{0}, \ldots, Q_{s}$ are quadratic functions of $x \in \Re^{n}$. Hence, condition (23) can be formulated as an LMI:

$\tilde{x}^{T}\left[\begin{array}{ll}Z_{0} & c_{0} \\ c_{0}^{T} & d_{0}\end{array}\right] \tilde{x} \geq \sum_{k=1}^{s} \delta_{k} \tilde{x}^{T}\left[\begin{array}{ll}Z_{k} & c_{k} \\ c_{k}^{T} & d_{k}\end{array}\right] \tilde{x}$

where $\tilde{x}=\left[\begin{array}{ll}x & 1\end{array}\right]^{T}$.

The great advantage of the above formulation is that the quadratic condition $\tilde{x}^{T}\left[\begin{array}{ll}Z_{0} & c_{0} \\ c_{0}^{T} & d_{0}\end{array}\right] \tilde{x} \geq 0$ should be satisfied in just a part of $\mathcal{F}$, meaning that the matrix $\left[\begin{array}{cc}Z_{0} & c_{0} \\ c_{0}^{T} & d_{0}\end{array}\right]$ is positive semi-definite. This rules out the possibility of a conservative formulation since at least all the quadratic conditions $\tilde{x}^{T}\left[\begin{array}{ll}Z_{k} & c_{k} \\ c_{k}^{T} & d_{k}\end{array}\right] \tilde{x} \geq 0, k \in I_{s}$ are fulfilled in a part of $\mathcal{F}$.

Remark 4: The substitution of condition (22) using Lemma may end up in a conservative LMI formulation. Nevertheless, in case of $s=1$, the converse is true on the condition that there is some $x$ to fulfill $Q_{1}(x)>0$. 
Remark 5: If we confine $Q_{k}(x)$ as $Q_{k}(x) \geq 0$, it is always theoretically possible to represent an arbitrary region $\Omega$ by a quadratic condition $Q_{k}(x) \geq 0$. However, in practice, the substitution is made in such a way that the states fulfilling all the conditions $Q_{k}(x) \geq 0, k=$ $1, \ldots, s$ transcend the original region $\Omega$. Condition (22) is more relaxed than condition (21). Even though in some cases, the conservative formulation may be found feasible, finding a more relaxed formulation is essential for searching a feasible LMI solution.

Remark 6: If the region $\Lambda_{q r}$ is to be represented (using the Lemma) as quadratic conditions $Q_{k}(x)=$ $0, k \in I_{s}$, the constraints $\delta_{k} \geq 0, k \in I_{s}$ can be cancelled as the Lemma holds regardless of the sign of $\delta_{k}, k \in I_{s}$.

The necessary step toward formulating LMI stability conditions is optimizing the parameters in the quadratic function (24) in such a way that the set of states fulfilling all $Q_{k}(x) \geq 0$ comprises the set $\Omega$. First, assume that the arbitrary region $\Omega$ encompassing the origin is given by a set of states, limited by two half-planes

$\left(f^{a}\right)^{T} \geq 0$ and $\left(f^{b}\right)^{T} \geq 0$

then the region $\Omega$ can be represented by

$x^{T} Z^{1} x \geq 0$, where $Z^{1}=f^{a}\left(f^{b}\right)^{T}+f^{b}\left(f^{a}\right)^{T}$.

The symmetrical property of the above condition is that both $x_{1}$ and $-x_{1}$ can fulfill the inequality (27). A region $\Omega$ can be substituted by quadratic inequalities (27) represented by only two hyperplanes on the condition that the dimension $n=2$. However, if the number of dimensions is greater than two $(n>2),(27)$ should be represented by more than two hyperplanes (all possible combinations of two different half-planes). This results in $\frac{\sigma(\sigma-1)}{2}$ different quadratic inequalities, where $\sigma$ is the number of half-planes. Since $x^{T} Z^{k} x \geq 0$ is true for all states, it is reasonable to eliminate the same combination of half-planes.

If the region $\Omega$ is limited by several half-planes, it is not possible for the region to be accurately represented by one quadratic inequality $x^{T} Z^{k} x \geq 0$, even considering the symmetric property, i.e. if $x \in \Omega \Rightarrow-x \in \Omega$. The set of states fulfilling $x^{T} Z^{1} x \geq 0$ for a quadratic inequality obtained by any combination of two half-planes representing $\Omega$ would be strictly larger than $\Omega$. In fact, there exist a number of suitable sets of states fulfilling the inequality $x^{T} Z_{1} x \geq 0$. Therefore, all acceptable combinations should be considered in order to identify the most suitable set. The variables $\delta^{k}$ are determined by solving the resulting LMI problem (25), if feasible. The quadratic inequality substituted by the region $\Omega$ is then known.
Now, in case the region $\Omega$ does not encompass the origin, the half-plane limiting the region is defined as

$f^{T} x+g \geq 0$,

which can be represented by the quadratic inequalities [4]:

$\tilde{x}^{T}\left[\begin{array}{cc}0 & f \\ f^{T} & 2 g\end{array}\right] \tilde{x}$

where ' 0 ' in the upper left corner represents an $n \times n$ zero matrix. If the region $\Omega$ is limited by a set given by more than two half-planes, as in the previous case, all possible combinations should be considered. To be more specific, if the half-planes are given by

$\left(f^{a}\right)^{T} x+g^{a} \geq 0$ and $\left(f^{b}\right)^{T} x+g^{b} \geq 0$,

then the resulting possible quadratic inequalities would be:

$\tilde{x}^{T}\left[\begin{array}{cc}f^{a}\left(f^{b}\right)^{T}+f^{b}\left(f^{a}\right)^{T} & g^{b} f^{a}+g^{a} f^{b} \\ g^{b}\left(f^{a}\right)^{T}+g^{a}\left(f^{b}\right)^{T} & 2 g^{a} g^{b}\end{array}\right] \tilde{x}$,

$\tilde{x}^{T}\left[\begin{array}{cc}0 & f^{a} \\ \left(f^{a}\right)^{T} & 2 g^{a}\end{array}\right] \tilde{x}$,

$\tilde{x}^{T}\left[\begin{array}{cc}0 & f^{b} \\ \left(f^{b}\right)^{T} & 2 g^{b}\end{array}\right] \tilde{x}$,

where $\tilde{x}=\left[\begin{array}{ll}x & 1\end{array}\right]^{T}$.

For the boundary region $\Lambda_{q r}$, which represents a hyperplane (or hypersurfaces), the substitute quadratic forms are defined as quadratic equalities as follows:

$f^{T} x+g=0$,

where $f=\left[f^{1} \ldots f^{n}\right]^{T} \in \Re^{n}$ and $g \in \Re$. The equivalent representation of (33) is

$2\left(\lambda^{T} x+\lambda^{n+1}\right)^{T}\left(f^{T} x+g\right)=0$,

where $\lambda=\left[\lambda^{1}, \ldots, \lambda^{n}\right]^{T} \in \Re^{n}$ and $\lambda^{n+1} \in \Re$ are arbitrary extra variables. The equality (34) can be alternatively stated as

$\tilde{x}^{T}\left[\begin{array}{c}\lambda \\ \lambda^{n+1}\end{array}\right]\left[\begin{array}{ll}f^{T} & g\end{array}\right] \tilde{x}+\tilde{x}^{T}\left[\begin{array}{l}f \\ g\end{array}\right]\left[\lambda^{T} \lambda^{n+1}\right] \tilde{x}=\sum_{k=1}^{n+1} \lambda^{k} \tilde{x}^{T} \tilde{Z}^{k} \tilde{x}=0$,

where

$\tilde{Z}^{k}=a^{k}\left[f^{T} g\right]+\left[f^{T} g\right]^{T}\left(a^{k}\right)^{T}$,

and $a^{k}$ is a column vector with $n$ elements such that

$e^{k}(i)=\left\{\begin{array}{l}1, i=k, \\ 0, i \neq k,\end{array}\right.$

where $i$ means the $i^{\text {th }}$ element of $a^{k}$. 


\subsection{LMI stability conditions}

The final step in investigating the stability of the grazing orbit is to formulate the stability theorem as LMIs. The stability analysis is based on the non-smooth TS fuzzy model of the hard-impact oscillator developed in section 3 .

Theorem 1: If there exist piecewise quadratic matrices $\tilde{P}_{q}, q \in I_{\Delta}$ and constants $\alpha, \beta, \mu_{k}^{q}, \nu_{k}^{q i j}, \eta_{k}^{q r}$, then the fixed point of the limit cycle is structurally stable in the sense of Lyapunov if there is a solution to $\min \beta$ subject to the following conditions:

$$
\begin{aligned}
& -\alpha>0, \beta>0, \mu_{k}^{q} \geq 0 \\
& -\left[\begin{array}{ll}
\alpha & 0 \\
0 & 0
\end{array}\right]+\sum_{k=1}^{s_{q}} \mu_{k}^{q}\left[\begin{array}{cc}
Z_{k}^{q} & c_{k}^{q} \\
\left(c_{k}^{q}\right)^{T} & d_{k}^{q}
\end{array}\right] \leq\left[\begin{array}{cc}
P_{q} & p_{q} \\
p_{q}^{T} & \pi_{q}
\end{array}\right], q \in I_{\Delta} \\
& -\left[\begin{array}{cc}
P_{q} & p_{q} \\
p_{q}^{T} & \pi_{q}
\end{array}\right] \leq\left[\begin{array}{ll}
\beta & 0 \\
0 & 0
\end{array}\right]+\sum_{k=1}^{s_{q}} \mu_{k}^{q}\left[\begin{array}{cc}
Z_{k}^{q} & c_{k}^{q} \\
\left(c_{k}^{q}\right)^{T} & d_{k}^{q}
\end{array}\right], q \in I_{\Delta} \\
& -(q, i, j) \in I_{\Omega}, \quad q \in I_{\Delta} \\
& {\left[\begin{array}{cc}
A^{j}\left(m_{i}\right) & B^{j}\left(m_{i}\right) \\
0 & 0
\end{array}\right]^{T}\left[\begin{array}{ll}
P_{q} & p_{q} \\
p_{q}^{T} & \pi_{q}
\end{array}\right]+\left[\begin{array}{cc}
P_{q} & p_{q} \\
p_{q}^{T} & \pi_{q}
\end{array}\right]} \\
& \times\left[\begin{array}{cc}
A^{j}\left(m_{i}\right) & B^{j}\left(m_{i}\right) \\
0 & 0
\end{array}\right]+\sum_{k=1}^{s_{q i j}} \nu_{k}^{q i j}\left[\begin{array}{cc}
Z_{k}^{q} & c_{k}^{q} \\
\left(c_{k}^{q}\right)^{T} & d_{k}^{q}
\end{array}\right] \leq 0 \\
& {\left[\begin{array}{cc}
J\left(m_{i}\right) & 0 \\
0 & 1
\end{array}\right]^{T}\left[\begin{array}{ll}
P_{r} & p_{r} \\
p_{r}^{T} & \pi_{r}
\end{array}\right]\left[\begin{array}{cc}
J\left(m_{i}\right) & 0 \\
0 & 1
\end{array}\right] \leq\left[\begin{array}{cc}
P_{q} & p_{q} \\
p_{q}^{T} & \pi_{q}
\end{array}\right]} \\
& +\left[\begin{array}{cr}
H\left(m_{i}\right) & 0 \\
0 & 1
\end{array}\right]^{T}\left[\begin{array}{cc}
P_{q} & p_{q} \\
p_{q}^{T} & \pi_{q}
\end{array}\right]\left[\begin{array}{cc}
H\left(m_{i}\right) & 0 \\
0 & 1
\end{array}\right] \\
& -\sum_{k=1}^{s_{q r}} \eta_{k}^{q r}\left[\begin{array}{cc}
Z_{k}^{q r} & c_{k}^{q r} \\
\left(c_{k}^{q r}\right)^{T} & d_{k}^{q r}
\end{array}\right],(q, r) \in I_{\Lambda}
\end{aligned}
$$

The proof of the above theorem is similar to that presented in the previous publication [27] except for the last LMI condition. The extension of the proof to include the complexity of discontinuous states when crossing the switching manifold is given in the Appendix.

An interesting feature of the above stability theorem, formulated as an LMI problem, is the ability to search for a feasible solution that yields a better estimate of exponential convergence [27]. Therefore, the feasibility of any solution is found through optimizing a minimum value of $\beta$. All the other variables $\alpha, \mu_{k}^{q}$, $\nu_{k}^{q i j}, \eta_{k}^{q r}$ and the matrices $P_{q}, p_{q}, \pi_{q}, q \in I_{\Delta}$ need to be determined by solving the above Theorem as an LMI (optimization) problem.

As illustrated in sections 2 and 3 , in the event of a grazing bifurcation, there is an abrupt change to a much larger chaotic orbit close to the grazing point arising from the stretching of the phase space $[8,11]$. Assuming the same parameters (see section 2) for the non-smooth
TS fuzzy model, the system loses its stability at an excitation amplitude of $0.2759 \mathrm{~N}$ to an unstable chaotic orbit as evident from Fig. 7a. However, if we change the parameter value of $m$ to an integer value, say 3 , the system preserves the structural stability of its local orbit, as seen in the bifurcation diagram of Fig. 7b. To verify the efficacy of the LMI formulation of Theorem 1 , it is used to predict the onset of the unstable chaotic behavior by investigating the exponential stability of the grazing limit cycle as an optimization problem.

Following the $\mathcal{S}$-procedure (see sections 4.1 and 4.2), the fuzzy state space is partitioned into regions $\Omega_{q}$ and $\Omega_{r}$, and a boundary region $\Lambda_{q r}$ which divides the two regions as it represents the discontinuity boundary $\Sigma_{i j}$. Solving the LMI problem for $m=2.97$ and a forcing function amplitude $F=0.2751 \mathrm{~N}$, yields the feasible solution:

$$
\begin{aligned}
\tilde{\mathbf{P}}_{\mathbf{1}}= & {\left[\begin{array}{ccc}
-422.546 & 6.7878 & -0.2307 \\
6.7878 & -335.691 & 0.2655 \\
-0.2307 & 0.2655 & -423.048
\end{array}\right], } \\
\tilde{\mathbf{P}}_{\mathbf{2}}= & {\left[\begin{array}{ccc}
7.1287 & 0.1781 & -2.7433 \\
0.1781 & 7.1287 & -3.3345 \\
-2.7433 & -3.3345 & -706.9402
\end{array}\right] . }
\end{aligned}
$$

with an optimum value of $\beta=193.3704$. This simply proves that the limit cycle of the system (12) is exponentially (structurally) stable as can be seen from Fig. 7 , where the local orbit is in the stable period- 1 region. Changing the parameters to $m=3$ and $F=1.4982 \mathrm{~N}$ also results in a feasible solution as above, proving the grazing orbit is stable. Applying Theorem 1 for any operating point in the chaotic region (from $F=0.2760 \mathrm{~N}$ to $F=0.2763 \mathrm{~N}, m=2.97$ ), cf. (Fig 7a), results in an infeasible solution, confirming the instability of the grazing orbit as expected after the grazing bifurcation.

As pointed out in the introduction, the existing approach for studying the stability of periodic solutions in impacting systems is zero-time discontinuity mapping (ZDM), in which a discrete-time stroboscopic Poincaré map is synthesized around the limit cycle in question. The Jacobian of the discrete map can then be employed to verify the stability of the limit cycle ${ }^{6}$. An outline including an example of this approach for hard-impact oscillators can be found in [11]. It is well-known that this approach suffers from the square-root singularity problem when studying the grazing orbit. Some of the Jacobian elements of the Poincaré map contain an inverse square-root function causing the Jacobian to assume infinite values near the grazing condition where the square-root terms approach zero $[11,28,29]$. To be

6 The system is stable if the multipliers of the Jacobian of the linearized discrete map lie inside the unit circle. 
more exact, the Jacobian of the zero-discontinuity map (ZDM) can be derived as:

$J_{Z D M^{7}}=I+\sqrt{2 a\left(x^{*}\right)} \frac{W\left(x^{*}\right) H_{x}}{2 \sqrt{-H_{\min }}}$,

where $H_{\min }$ is the minimum value of $H(\varphi(x))$ at the grazing point, $H_{x}=\frac{\partial H(x)}{\partial x}$ and $a\left(x^{*}\right)$ is the acceleration of the flow $\varphi$ at the grazing point ${ }^{8}$ (Fig. 2b). The term $\sqrt{-H_{\min }}$ explains the square-root singularity problem already discussed.

Using our approach, the fuzzy state space should be divided into finer regions in order to find a solution near grazing. For example, if we try four region partitions, the discontinuous Lyapunov function (19) is then defined as $V(x)=V_{q}(x),\left(x, m_{i}\right) \in \Omega_{q}, q=1,2,3,4, i=$ 1,2 . This further partitioning actually reduces the possibility of a conservative LMI formulation very near the grazing point ( $m=2.97$ and $F=0.2757 \mathrm{~N})$ resulting in a feasible solution as given below with an optimum value of $\beta=241.29$ :

$$
\begin{gathered}
\tilde{\mathbf{P}}_{\mathbf{1}}=\left[\begin{array}{ccc}
-422.4312 & 8.7087 & -0.2833 \\
8.7087 & -335.7263 & 0.3126 \\
-0.2833 & 0.3126 & -422.9401
\end{array}\right], \\
\tilde{\mathbf{P}}_{\mathbf{2}}=\left[\begin{array}{ccc}
9.2904 & 0.2321 & -4.3017 \\
0.2321 & 9.2904 & -4.3548 \\
-4.3017 & -4.3548 & -706.8930
\end{array}\right], \\
\tilde{\mathbf{P}}_{\mathbf{3}}=\left[\begin{array}{ccc}
6.7425 & 0.1684 & -3.4002 \\
0.1684 & 6.7425 & -3.5557 \\
-3.4002 & -0.3557 & -706.9479
\end{array}\right], \\
\tilde{\mathbf{P}}_{\mathbf{4}}=\left[\begin{array}{ccc}
-422.1627 & 8.4460 & -0.2702 \\
8.4460 & -335.6612 & 0.3301 \\
-0.2702 & 0.3301 & -423.0231
\end{array}\right] .
\end{gathered}
$$

The above matrices demonstrate how the problem of infinite stretching of the phase space near the grazing event can be overcome by finer partitioning when applying the proposed LMI stability conditions in Theorem 1.

\section{Conclusion}

A novel TS fuzzy formalism for modeling impact-oscillator systems is suggested. The proposed modeling structure is synthesized to include the dynamics of discontinuous (state) jumps when crossing the switching manifold and take into account the unique nonlinearities which characterize impacting dynamics close to the grazing event. It has been shown that the non-smooth TS fuzzy model can well represent all the observed nonlinear behaviors in impacting systems including all grazing dynamical events.

\footnotetext{
8 for a complete derivation of $J_{Z D M}$ please refer to [11].
}

Based on the proposed non-smooth TS fuzzy model, a Lyapunov framework is further proposed for studying the stability of the grazing orbit. Non-smooth Lyapunov theory, as a natural choice for non-smooth model-based analysis is employed and formulated as linear matrix inequality (LMI) conditions to be solved by numerical interior-point methods. The stability conditions have been shown to be able to accurately predict the onset of the sudden change to a large chaotic attractor, induced by a grazing event. The analysis provides a new tool for the qualitative study of hard impacting systems. Using readily-available convex optimization solvers, the square-root singularity problem near the grazing event associated with the discrete-mapping approach can be avoided, allowing the onset of the grazing bifurcation to be located.

\section{Appendix}

Proof of Theorem 1: It's already known that the discontinuity boundary $\Sigma$ is the zero set of the smooth function $H$. If we assume the set $\partial S_{1}^{+}=\left\{x: \frac{\partial H(x)}{\partial x_{1}}\right\}$ and the function $\zeta \in \partial S_{1}^{+}$, which is defined as $\zeta: x \mapsto r x$, then the last condition of stability can be written in the sense of Lyapunov as:

$\left(x, m_{i}\right) \in \Lambda_{q r}, \quad V_{r}\left(\chi\left(x, m_{i}\right)\right) \leq V_{q}(x)+V\left(\zeta\left(x, m_{i}\right)\right), \quad(q, r) \in I_{\Lambda}$.

Following the procedure stated in section 4 , the above condition can be recast on LMI as given in Theorem 1.

Since the continuous fuzzy states $\left(x, m_{i}\right), i=1,2, \ldots, N$ can become discontinuous without passing to another state space fuzzy region $\Omega_{q}$ and without changing to the next discrete state $m_{i}$, the switching manifold can be represented as the region $\Lambda_{q q}$ for such states. Moreover, due to state discontinuities, if $\Lambda_{q r} \neq 0, \Omega_{q}$ must not be a neighboring set to $\Omega_{r}$.

It is necessary to assume that for all the conditions in the theorem, there is a finite number of discontinuous states in finite time. This implies that the states defined by the function $\chi$ (Remark 2), cannot undergo consecutive discontinuous jump in an infinite manner.

\section{References}

1. Adly, S.: Nonsmooth Dynamical Systems: an overview. In: International Workshop on Resonance Oscillations and Stability of Nonsmooth Systems (2009)

2. Bergsten, P.: Observers and controllers for takagi-sugeno fuzzy systems. Ph.D. thesis, Örebro (2001)

3. Bishop, S.: Impact oscillators. Philosophical Transactions: Physical Sciences and Engineering 347(1683), 347351 (1994)

4. Boyd, S., Ghaoui, L.E., Feron, E., Balakrishnan, V.: Linear Matrix Inequalities in System and Control Theory. SIAM, Philadelphia (1994) 
5. Branicky, M.S.: Stability of switched and hybrid systems. In: Proceedings of the 33rd IEEE Conference on Decision and Control, pp. 3498-3503. Lake Buena Vista, FL (1994)

6. Chen, B., Tseng, C., Uang, H.J.: Mixed $H_{2} / H_{\infty}$ fuzzy output feedback control design for nonlinear dynamic systems: an LMI approach. IEEE Transactions on Fuzzy Systems 8(3) (2000)

7. Chin, W., Ott, E., Nusse, H., Grebogi, C.: Universal behavior of impact oscillators near grazing incidence. Physics Letters A 201(2-3), 197-204 (1995)

8. Dankowicz, H., Svahn, F.: On the stabilizability of neargrazing dynamics in impact oscillators. International Journal of Robust and Nonlinear Control 17(15), 1405$1429(2007)$

9. DeCarlo, R., Peleties, P.: Asymptotic Stability of mswitched systems using Lyapunov-like functions. In: Proceedings of American Control Coneference, pp. 16791684. Boston, MA (1991)

10. Dercole, F., Kuznetsov, Y.: SlideCont: An Auto 97 driver for bifurcation analysis of Filippov systems. ACM Transactions on Mathematical Software 31(1), 95-119 (2005)

11. Di Bernardo, M., Budd, C., Kowalczyk, P., Champneys, A.: Piecewise-Smooth Dynamical Systems: Theory And Applications. Springer (2007)

12. Ervin, E., Wickert, J.: Experiments on a beam-rigid body structure repetitively impacting a rod. Nonlinear Dynamics 50(3), 701-716 (2007)

13. Filippov, A.F.: Differential equations with discontinuous righthand sides. Kluwer Academic Publishers, Dortrecht (1988)

14. Ing, J., Pavlovskaia, E., Wiercigroch, M.: Dynamics of a nearly symmetrical piecewise linear oscillator close to grazing incidence: Modelling and experimental verification. Nonlinear Dynamics 46(3), 225-238 (2006)

15. Ing, J., Pavlovskaia, E., Wiercigroch, M., Banerjee, S.: Experimental study of impact oscillator with one-sided elastic constraint. philosophical transactions-royal society of london series a mathematical physical and engineering sciences 366(1866), 679 (2008)

16. Johansson, M., Rantzer, A.: Computation of piecewise quadratic Lyapunov functions for hybrid systems. IEEE Transactions on Automatic Control 43(4), 555-559 (1998)

17. Johansson, M., Rantzer, A., Årzén, K.: Piecewise quadratic stability of fuzzy systems. IEEE Transaction on Fuzzy Systems 7(6) (1999)

18. Kundu, S., Banerjee, S., Giaouris, D.: Vanishing singularity in hard impacting systems. Discrete and Continuous Dynamical Systems - Series B (2010, under submission)

19. Lee, H., Park, J., Chen, G.: Robust fuzzy control of nonlinear systems with parametric uncertainties. IEEE transactions on fuzzy systems 9(2), 369-379 (2001)

20. Leine, R.I., Nijmeijer, H.: Dynamics and Bifurcations of Non-Smooth Mechanical Systems. Springer, Berlin, Germany (2004)

21. Lenci, S., Rega, G.: A procedure for reducing the chaotic response region in an impact mechanical system. Nonlinear Dynamics 15(4), 391-409 (1998)

22. Li, Z.: Fuzzy chaotic systems: modeling, control, and applications. Springer (2006)

23. M. Doĝruel, U. Özgüner: Stability of hybrid systems. In: IEEE International Symposium on Intelligent Control, pp. 129-134. Coloumbos, OH (1994)

24. Ma, Y., Agarwal, M., Banerjee, S.: Border collision bifurcations in a soft impact system. Physics Letters A 354(4), 281-287 (2006)
25. Ma, Y., Ing, J., Banerjee, S., Wiercigroch, M., Pavlovskaia, E.: The nature of the normal form map for soft impacting systems. International Journal of NonLinear Mechanics 43(6), 504-513 (2008)

26. Mehran, K., Giaouris, D., Zahawi, B.: Modeling and Stability Analysis of Closed Loop Current-Mode Controlled Ćuk Converter using Takagi-Sugeno Fuzzy Approach. In: CHAOS'09. IFAC, England, London (2009)

27. Mehran, K., Giaouris, D., Zahawi, B.: Stability analysis and control of nonlinear phenamena in boost converter using model-based takagi-sugeno fuzzy approach. IEEE Transactions in Circuits and Systems - I 57(1), 200-212 (2010)

28. Nordmark, A.: Non-periodic motion caused by grazing incidence in an impact oscillator. Journal of Sound Vibration 145, 279-297 (1991)

29. Nordmark, A.: Universal limit mapping in grazing bifurcations. Physical review E 55(1), 266-270 (1997)

30. Nordmark, A., Piiroinen, P.: Simulation and stability analysis of impacting systems with complete chattering. Nonlinear Dynamics 58(1), 85-106 (2009)

31. Nusse, H., Ott, E., Yorke, J.: Border-collision bifurcations: An explanation for observed bifurcation phenomena. Physical Review E 49(2), 1073-1076 (1994)

32. Santos, I.: Modeling and numerical study of nonsmooth dynamical systems. Ph.D. thesis, Universitat Politécnica de Catalunya, Barcelona (2006)

33. Shaw, S., Holmes, P.: A periodically forced piecewise linear oscillator. Journal of Sound and Vibration 90(1), 129-155 (1983)

34. Shevitz, D., Paden, B.: Lyapunov stability theory of nonsmooth systems. IEEE Transactions on Automatic Control 39(9), 1910-1914 (1994)

35. Takagi, T., Sugeno, M.: Fuzzy identification of systems and its applications to modeling and control. IEEE Transaction on Systems, Man and Cybernetics 15(1) 116-132 (1985)

36. Tanaka, K.: Fuzzy Control Systems Design and Analysis: A Linear Matrix Inequality Approach. John Wiley \& Sons, Newark (2001)

37. Tanaka, K., Ikeda, T., Wang, H.: A unified approach to controlling chaos via an LMI-based fuzzy control system design. IEEE Transactions on Circuits and Systems I: Fundamental Theory and Applications 45(10), 1021$1040(1998)$

38. Tanaka, K., Ikeda, T., Wang, H.: Fuzzy regulators and fuzzy observers: relaxed stability conditions and LMIbased designs. IEEE Transactions on Fuzzy Systems 6(2) (1998)

39. Tanaka, K., Sugeno, M.: Stability analysis and design of fuzzy control systems. Fuzzy sets and systems 45, 135$156(1992)$

40. Ting, C.S.: Stability analysis and design of TakagiSugeno fuzzy systems. Information Sciences 176(19), 2817-2845 (2006)

41. Tuan, H.D., Apkarian, P., Narikiyo, T., Kanota, M.: New fuzzy control model and dynamic output feedback parallel distributed compensation. IEEE Transactions on Fuzzy Systems 12(1) (2004)

42. Wagg, D., Karpodinis, G., Bishop, S.: An experimental study of the impulse response of a vibro-impacting cantilever beam. Journal of Sound and Vibration 228(2), 243-264 (1999)

43. Wiercigroch, M., Sin, V.: Experimental study of a symmetrical piecewise base-excited oscillator. Journal of Applied Mechanics 65, 657 (1998) 